\title{
AGB nucleosynthesis in the Large Magellanic Cloud ${ }^{\star}$ Detailed abundance analysis of the RV Tauri star MACHO 47.2496.8
}

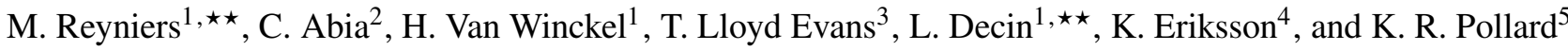 \\ ${ }^{1}$ Instituut voor Sterrenkunde, Departement Natuurkunde en Sterrenkunde, K.U. Leuven, Celestijnenlaan 200D, 3001 Leuven, \\ Belgium \\ e-mail: maarten@ster.kuleuven.be \\ 2 Dpto. Física Teórica y del Cosmos, Universidad de Granada, 18071 Granada, Spain \\ 3 SUPA, School of Physics and Astronomy, University of St. Andrews, North Haugh, St. Andrews, Fife KY16 9SS, Scotland, UK \\ 4 Department of Astronomy and Space Physics, Uppsala University, Box 515, 75120 Uppsala, Sweden \\ 5 Department of Physics and Astronomy, University of Canterbury, Private Bag 4800, Christchurch, New Zealand
}

Received 23 June 2006 / Accepted 2 October 2006

\section{ABSTRACT}

\begin{abstract}
Context. Abundance analysis of post-AGB objects as probes of AGB nucleosynthesis.
Aims. A detailed photospheric abundance study is performed on the carbon-rich post-AGB candidate MACHO 47.2496.8 in the LMC. Methods. High-resolution, high signal-to-noise ESO VLT-UVES spectra of MACHO 47.2496.8 are analysed by performing detailed spectrum synthesis modelling using state-of-the-art carbon-rich MARCS atmosphere models.

Results. The spectrum of MACHO 47.2496.8 is not only dominated by bands of carbon bearing molecules, but also by lines of atomic transitions of s-process elements. The metallicity of $[\mathrm{Fe} / \mathrm{H}]=-1.4$ is surprisingly low for a field $\mathrm{LMC}$ star. The $\mathrm{C} / \mathrm{O}$ ratio, however difficult to quantify, is greater than 2, and the s-process enrichment is large: the light s-process elements are enhanced by 1.2 dex compared to iron $([\mathrm{ls} / \mathrm{Fe}]=+1.2)$, while for the heavy s-process elements an even stronger enrichment is measured: $[\mathrm{hs} / \mathrm{Fe}]=+2.1$. The lead abundance is comparable to the [hs/Fe]. With its low intrinsic metallicity and its luminosity at the low end of the carbon star luminosity function, the star represents likely the final stage of a low initial mass star.

Conclusions. The LMC RV Tauri star MACHO 47.2496.8 is highly carbon and s-process enriched, and is most probable a genuine post-C(N-type) AGB star. This is the first detailed abundance analysis of an extragalactic post-AGB star to date.
\end{abstract}

Key words. stars: AGB and post-AGB - stars: abundances - stars: carbon - stars: individual: MACHO 47.2496.8 Magellanic Clouds

\section{Introduction}

It is well-known that the MACHO experiment towards the Large Magellanic Cloud (LMC) yielded invaluable contributions to variable star research. The discovery of five distinct periodluminosity (PL) relations of lower mass RGB and AGB giants (e.g. Wood et al. 1999) is probably the best known example. Another legacy of the experiment, was the discovery of 33 Pop II Cepheids and RV Tauri stars in the LMC (Alcock et al. 1998) and the definition of a single PL relation of both groups with the appearance of the RV Tauri characteristic lightcurves (alternating deep and shallow minima) at the high luminosity end: the RV Tauri stars are a direct extension of Pop II Cepheids to longer periods. Absolute luminosities were derived and the RV Tauri stars were confirmed to be likely post-AGB stars, making these objects members of the small group of post-AGB stars with known luminosities. For Galactic RV Tauri stars there is no direct probe to the luminosity but they were identified as postAGB stars by Jura (1986), mainly on the basis of the presence of an IR excess due to circumstellar dust around many of them.

The post-AGB phase is a short phase, so not many of these sources are known (see Stasińska et al. 2006, for a catalogue

\footnotetext{
* Based on observations collected at the European Southern Observatory, Chile (programme 074.D-0619(A)).

$\star \star$ Postdoctoral fellow of the Fund for Scientific Research, Flanders.
}

of candidate post-AGB objects). Their temperature-gravity domain makes it possible to study a very wide range in chemical species by their atomic transitions (if optically bright enough). They are therefore ideal objects to constrain the AGB nucleosynthetic and evolutionary models. Interestingly, post-AGB stars are chemically much more diverse than theoretically anticipated: only a very small group of objects shows direct chemical evidence for AGB nucleosynthesis, being enhanced in carbon and enhanced in neutron capture s-process elements (e.g. review by Van Winckel 2003, and references therein). Since the post-AGB evolutionary tracks pass the Cepheid instability strip, the pulsating RV Tauri stars could be, in principle, good candidates to test further AGB chemical evolutionary models.

In recent years it became, however, clear that also for RV Tauri stars the chemical picture is complex and chemical evidence for a post-AGB nature (possible C-enrichement and sprocess overabundances) is not found in Galactic RV Tauri stars (except for maybe V453 Oph, for which a mild s-process overabundance was found, see Deroo et al. 2005). In RV Tauri stars, depletion abundance patterns prevail (Giridhar et al. 2005; Maas et al. 2005). The basic scenario of the badly understood depletion process involves a chemical fractionation due to dust formation in the circumstellar environment followed by a decoupling of the gas and the dust with a reaccretion of the cleaned gas on the stellar photosphere, which leaves it depleted of the refractory 
Table 1. Basic parameters of MACHO 47.2496.8.

\begin{tabular}{rrr}
\hline \hline \multicolumn{2}{c}{ MACHO 47.2496.8 } \\
\hline Coordinates & $\alpha_{2000}$ & 45543.23 \\
& $\delta_{2000}$ & -675110.4 \\
\hline Mean magnitude & $V$ & 14.97 \\
\hline Spectral type & & Supergiant \\
& & pulsating between \\
& & early K and late F \\
\hline
\end{tabular}

Source coordinates: MACHO; magnitude: Alcock et al. (1998); spectral type: Pollard \& Lloyd Evans (2000).

elements. Waters et al. (1992) showed that the most favourable circumstance for this process to occur is, if the circumstellar dust is trapped in a disc. The presence of a disc in evolved objects is likely to be related to binarity (e.g. Van Winckel 2003) which means that binarity must be widespread in RV Tauri stars (see also De Ruyter et al. 2006).

The LMC sample of RV Tauri stars with their known luminosity is a unique sample to study the nature of these stars and of the post-AGB evolution in general. Moreover, abundance analyses of post-3rd dredge-up stars in the LMC should make it possible to study the yields of the AGB nucleosynthesis in a more metal deficient environment than the Galaxy. With the advent of high-resolution spectrographs on $8 \mathrm{~m}$ class telescopes, it is now feasible to study in detail the abundance patterns of these individual RV Tauri candidates in the LMC. The brightest object of the Alcock et al. (1998) sample, MACHO*04:55:43.267:51:10, also named as MACHO 47.2496.8, was studied at low resolution by Pollard \& Lloyd Evans (2000) and by Lloyd Evans \& Pollard (2004). The basic parameters of MACHO 47.2496.8 are summarised in Table 1. Contrary to what is found in Galactic RV Tauri stars, this object shows clear indications of a strong C-enhancement and of s-process overabundances. The low-resolution spectra at different photometric phases of MACHO 47.2496.8 show that it is strongly carbon enriched $(\mathrm{C} / \mathrm{O}>1)$ with strong $\mathrm{C}_{2}$ bands at deep minima. Spectra taken at a resolution of $0.12 \mathrm{~nm}$ with the $1.9 \mathrm{~m}$ SAAO telescope around deep minimum (phase 0.88) and with the Anglo-Australian Telescope near maximum light (phase 0.61) show that the $C_{2}$ bands disappear almost completely at maximum light, with only the actual bandhead of the origin band at $5165 \AA$ still readily detectable. The spectrum at minimum showed strong bandheads of ${ }^{12} \mathrm{C}^{12} \mathrm{C}$ at 4737 and $4715 \AA$ but the $4744 \AA$ bandhead of ${ }^{12} \mathrm{C}^{13} \mathrm{C}$ was very weak or absent, indicative of a high ${ }^{12} \mathrm{C} /{ }^{13} \mathrm{C}$ ratio. Enhancements of the Ba II spectral features at $4554 \AA$ and $4934 \AA$ were detected on these spectra, making this object a very interesting star to study the AGB nucleosynthesis in the LMC in detail.

In this paper, we will present a detailed abundance analysis based on high-resolution, high signal-to-noise VLT-UVES spectra. The paper is organised as follows: in Sect. 2 we summarise the available photometry of the object, and discuss its variability and extinction. In Sect. 3, the high-resolution observations are briefly discussed, while Sect. 4 is devoted to the actual analysis and the abundance results. In Sect. 5 we discuss the abundance pattern, and address the question whether the star is intrinsically or extrinsically enriched in s-process elements. We end with the main conclusions (Sect. 6).

\section{Variability, extinction and luminosity}

\subsection{MACHO photometry}

The database of the MACHO project (Alcock et al. 1992, 1995), accessible via http: //wwwmacho. anu.edu.au/, contains 260 simultaneous measurements of MACHO 47.2496.8 in the blue MACHO filter $V_{\mathrm{M}}$ and red MACHO filter $R_{\mathrm{M}}$, and 465 additional measurements in $V_{\mathrm{M}}$ only. We performed a frequency analysis on the $725 V_{\mathrm{M}}$ magnitudes, using the Jurkevich-Stellingwerf phase dispersion minimization (PDM) method (Stellingwerf 1978), and deduced a "formal" period (the period between two successive deep minima) of $P=112.97 \mathrm{~d}$ ( $\theta$-statistic of $\theta=0.23)$, which is slightly longer than the period in Alcock et al. (1998), being $P=112.45 \mathrm{~d}$. Two outliers were removed from the frequency analysis. A part of the $V_{\mathrm{M}}$ light curve for MACHO 47.2496.8 is also shown in Fig. 1 of Pollard \& Lloyd Evans (2000). Our period of 112.97 days and the epoch of minimum light, JD 2448673.384 , are used as the basis for the phases of observations reported here.

A realistic error estimate on a period determination is always difficult to obtain. Here, we use a rather straightforward method to give a rough error estimate on the period: the width of the PDM minimum is a measure for the spread on the "acceptable" periods, i.e. the periods for which the corresponding phase diagrams are still acceptable. This width is $\sim 3 \times 10^{-5} \mathrm{c} / \mathrm{d}$ in frequency space, resulting in an uncertainty of $\pm 0.2 \mathrm{~d}$ on the period. As an extra check, we performed an additional frequency analysis on the $260 V_{\mathrm{M}}-R_{\mathrm{M}}$ colour data points. This analysis yielded a slightly shorter period $(P=112.86 \mathrm{~d})$, but still within the quoted error of $\pm 0.2 \mathrm{~d}$.

The conversion of the raw MACHO magnitudes to a standard photometric system is not straightforward, since the two filters are very broad, and they are only partly overlapping with the classical Johnson or Kron-Cousins filters (see Fig. 1 of Alcock et al. 1999, for the filter pass bands). The conversion to the standard Johnson-Kron-Cousins system is performed using the formulae (1), (2), (5) and (6) of Alcock et al. (1999). In a first step, only the observations that are taken simultaneously in both bands, were calibrated to the Johnson-Kron-Cousins system, since $V_{\mathrm{M}}-R_{\mathrm{M}}$ is needed to perform the conversion. Then, the calibration was extended to all $725 V_{\mathrm{M}}$ points, using a calibration relation that was inferred from the calibration of the 260 simultaneous points. A phase diagram of the converted $V$ magnitude, and the converted $V-R$ colour, is shown in Fig. 1.

From Fig. 1 it is clear that the phase diagram displays a large dispersion around the mean curve, especially around the two minima. This dispersion is caused by cycle-to-cycle variations. Note that the colour variations are much more stable over the covered cycles. Such cycle-to-cycle behaviour in light and colour curves is often observed in RV Tauri variables (e.g. Pollard et al. 1996).

\subsection{Near-infared photometry}

Near-infrared photometry was obtained with the Mk III Infrared Photometer and chopping secondary at the Cassegrain focus of the $1.9 \mathrm{~m}$ reflector of the South African Astronomical Observatory by one of us (KRP). This photometry was complemented by near-IR survey photometry of 2MASS and DENIS. All near-IR data, together with their JD and phase, are gathered in Table 2. 


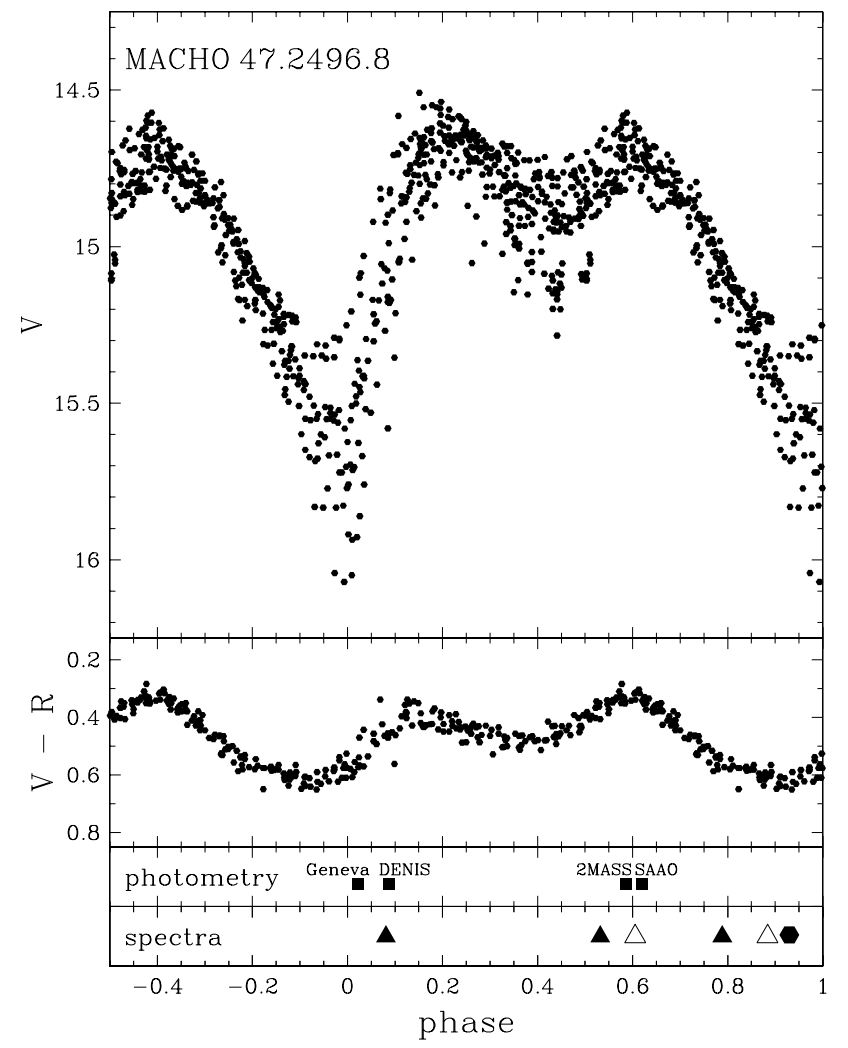

Fig. 1. The MACHO $V$ and $V-R$ phase diagram, converted to the standard Johnson $V$ and Kron-Cousins $R$, constructed with a period of 112.97 days, and a zero phase at deep minimum at JD 2448673.384. For the $V-R$ colour diagram, only the simultaneous measurements in both bands are shown. At the bottom, the phases of the photometric and spectroscopic data are given. The symbols of the spectroscopic data are as follows: the full triangles are low resolution spectra presented in Pollard \& Lloyd Evans (2000); the open ones are discussed in Lloyd Evans \& Pollard (2004); the phase of the UVES spectrum (0.93) is shown with a hexagon.

\subsection{Optical CCD photometry with Euler}

Optical CCD photometry in five different bands was collected with the C2 camera at the $1.2 \mathrm{~m}$ Euler telescope on La Silla on 23/24 January, 2006. The filters that were used are the Geneva $U, B$ and $V$, completed with a Gunn- $R$ and a Cousins- $I$ filter. Absolute photometry was derived by using exposures of the standard stars HD 47645 and SAO 131547 that were taken immediately before and after the MACHO 47.2496.8 frames. This absolute calibration was cross-checked by the extraction of two stars on the LMC-frame with published photometry which are situated less than 4' from MACHO 47.2496.8: CSI67-045523 (filters $U, B$ and $V$, Schmidt-Kaler et al. 1999) and MACHO 47.2496.19 (filters $V$ and $R$, Keller et al. 2002). Taking into account that there are several transformation formulae involved in the conversion of the Geneva and Gunn filters to the Johnson-Cousins system (Schombert et al. 1990; Harmanec \& Božić 2001), the magnitudes of the two check-stars are very close to the published ones.

Since there is a nearby star at approx. $2^{\prime \prime}$, the extraction of MACHO 47.2496.8 was by no means easy. We corrected the pixels of our target that are affected by this nearby star by their point symmetric counterparts on the unaffected side of the photocenter. This induces an extra uncertainty in the derived magnitudes, but, since the corrected pixels are situated at the boundary of the psf, this uncertainty turned out to be not larger than
Table 2. Optical Geneva and near-IR photometry of MACHO 47.2496.8, together with their JD and phase. The Geneva $R$ and $I$ are from the Gunn resp. Cousins photometric systems.

\begin{tabular}{|c|c|c|c|c|c|c|}
\hline$\overline{\mathrm{JD}}$ & Phase & $\bar{U}$ & $\begin{array}{l}B \\
\end{array}$ & $\bar{V}$ & $\bar{R}$ & $I$ \\
\hline \multicolumn{7}{|c|}{ Geneva } \\
\hline 2453759.6 & 0.02 & 19.15 & 16.81 & 15.94 & 15.18 & 14.66 \\
\hline JD & Phase & $I$ & $J$ & $H$ & $K$ & \\
\hline \multicolumn{7}{|c|}{ SAAO } \\
\hline 2450437.4 & 0.62 & & 13.18 & 12.67 & 12.54 & \\
\hline 2450438.3 & 0.62 & & 13.20 & 12.56 & 12.29 & \\
\hline Mean & 0.62 & & 13.19 & 12.61 & 12.41 & \\
\hline$\sigma$ & & & .04 & .04 & .05 & \\
\hline \multicolumn{7}{|c|}{$2 \mathrm{MASS}$} \\
\hline 2451111.8 & 0.58 & & 13.12 & 12.71 & 12.51 & \\
\hline$\sigma$ & & & .03 & .04 & .04 & \\
\hline \multicolumn{7}{|c|}{ DENIS } \\
\hline 2450377.8 & 0.09 & 14.02 & 13.22 & & 12.53 & \\
\hline$\sigma$ & & .03 & .08 & & .13 & \\
\hline
\end{tabular}

The Geneva $R$ can be converted to $R_{\mathrm{C}}$, the Cousins $R$ magnitude, by the conversion forumula from Schombert et al. (1990), yielding $R_{\mathrm{C}}=$ 14.89 .

$0.05 \mathrm{mag}$. The photometry is given in Table 2 . The phase of the Euler observations, 0.02 , has an estimated error of $\sim 0.07$, due to the quite large time gap between the MACHO photometry (on which the period is based) and the C2-Euler observations. Note that the MACHO photometry is not affected by this nearby star, since the latter star has its own MACHO identification: MACHO 47.2496.21.

\subsection{Spectral energy distribution}

The photometry from Table 2 was used to construct a Spectral Energy Distribution of MACHO 47.2496.8. The total reddening $E(B-V)$ was determined by minimising the difference between the magnitudes and a reddened model atmosphere. For this model atmosphere, we used the parameters that were determined in our abundance analysis (Sect. 4.2). Note that the optical Geneva photometry and the UVES spectrum from which the model parameters are derived, differ less than 0.1 in phase. Such a small phase difference is expected to result in only a small shift in atmospheric parameters. The result can be found in Fig. 2; an $E(B-V)=0.44$ was found. Due to the presence of the innumerable spectral lines in the model atmosphere, the specific passbands of the filters used were taken into account (Geneva system: http: //obswww . unige.ch/, DENIS: Fouqué et al. 2000, SAAO: Glass 1973). The integration of the scaled model combined with an LMC distance of $50 \mathrm{kpc}$, yields a luminosity of $\sim 5000 L_{\odot}\left(M_{\text {bol }} \simeq-4.5\right)$.

A specific problem arose for the $I$ magnitude. The Cousins $I$ taken with Euler turned out to be suspiciously low. The Cousins $I$ was the only magnitude that could not be checked with a star on the CCD frame itself (see Sect. 2.3). The DENIS-I magnitude - in gray on Fig. 2 - could not be reconciled with the other optical photometry as well, possibly attributable to a phase shift. We did not take either I magnitude into account in the minimisation.

We made an additional SED with the MACHO photometry, confined to those measurements with a a phase close to the UVES phase (difference $<0.05$ ), since the model atmosphere parameters are based on the UVES spectrum. The SED made with the MACHO $V$ and $R$, completed with the DENIS $I$ and the nearIR $J H K$ measurements, yielded a reddening of $E(B-V)=0.33$ and integration of the scaled model yielded a total luminosity of 


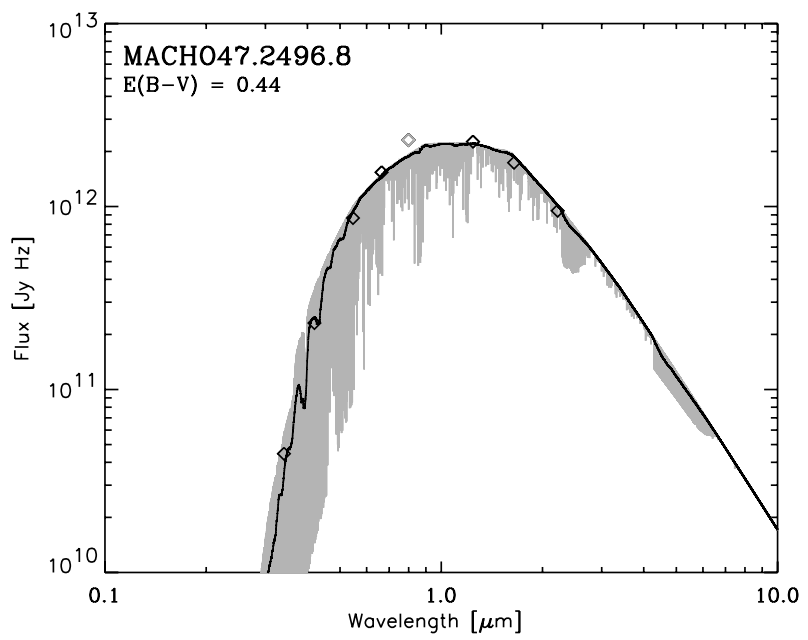

Fig. 2. The spectral energy distribution (SED) of MACHO 47.2496.8. Diamonds are the measured magnitudes (converted to fluxes): Geneva $U, B, V$ and Cousins $R$ taken with C2+Euler, $I$ from DENIS (in gray), and SAAO $J, H, K$ (see also Table 2). The MARCS model is shown in gray, while a smoothed version is shown in a full black line. The minimisation is made using the unsmoothed model in combination with the specific passbands of the photometric filters involved. The $I$ magnitude could not be fitted, and is excluded in the minimisation. Possibly a phase difference is causing this discrepancy.

$\sim 4600 L_{\odot}\left(M_{\text {bol }} \simeq-4.4\right)$. The fit is, however, of a lower quality than the fit in Fig. 2, hence we take the value of $5000 L_{\odot}$ as the final value for the luminosity of MACHO 47.2496.8.

\subsection{Interstellar extinction}

The reddening derived in Sect. 2.4, is the total reddening, and can have, besides the interstellar component, a circumstellar component as well. The interstellar extinction towards the LMC is well studied in literature. $U B V$ photometry and spectral types of $450 \mathrm{OB}$ stars in the western half of the LMC (Isserstedt 1975, 1979, 1982; Ardeberg et al. 1972; Rousseau et al. 1978; Crampton 1979; Conti et al. 1986; Fitzpatrick 1988) give a mean $E(B-V)$ of $0.13 \mathrm{mag}$, using the method and calibrations of Fitzpatrick \& Garmany (1990). There are larger values in OB associations and a region of lower values in the North of the LMC. The region surrounding MACHO 47.2496.8 appears fairly uniform on the Digitised Sky Survey, and the mean reddening of 16 stars within 30 arcmin is $0.11 \mathrm{mag}$. However Zaritsky et al. (2004) find that the distribution of reddening values for cool stars differs from that of hot stars and is bimodal, such that stars have $E(B-V)$ near zero or near $0.15 \mathrm{mag}$, depending on whether they lie in front of or behind a widespread thin dust layer. We cannot place MACHO 47.2496.8 or other carbon stars relative to this dust layer and, therefore, have to assume that the reddening is not significantly different from the average for carbon stars.

\section{High resolution observations}

High resolution, high signal-to-noise optical spectra of our programme star were taken with the UVES spectrograph mounted on the VLT-UT2 (Kueyen) telescope, in the program to obtain high-quality data of a larger sample of post-AGB objects. The data were acquired in visitor mode during ESO period \#74 by one of us (HVW). These observations fit in the framework of our ongoing program to study the photospheric chemical composition of stars in their last stages of evolution
Table 3. Log of the high-resolution VLT-UVES observations. Small spectral gaps occur between $5757 \AA$ and $5833 \AA$ and between $8521 \AA$ and $8660 \AA$ due to the spatial gap between the two UVES CCDs. Since continuum spectral intervals are hardly found in the spectrum, the signal-to-noise ratios $S / N$ given in the rightmost column, are only indicative, and should be interpreted more as lower limits than as fixed values.

\begin{tabular}{ccccc}
\hline \hline Date & $\begin{array}{c}\text { UT } \\
\text { start }\end{array}$ & $\begin{array}{c}\text { Exp.time } \\
(\mathrm{s})\end{array}$ & $\begin{array}{c}\text { Wavelength } \\
\text { interval }(\AA)\end{array}$ & $S / N$ \\
\hline \multicolumn{5}{c}{ VLT-UT2 + UVES } \\
\hline $2005-02-08$ & $02: 49$ & 7200 & $4780-6808$ & 70 \\
$2005-02-09$ & $00: 30$ & 7200 & $3758-4983$ & 40 \\
$2005-02-09$ & $00: 30$ & 7200 & $6705-10084$ & 80 \\
\hline
\end{tabular}

(e.g. Reyniers et al. 2004; Deroo et al. 2005). The resolving power of the UVES spectra varies between $~ 60000$ and $\sim 65000$. Spectra were taken with the chip unbinned. The spectral interval covered and some other details about the observations are given in Table 3. Since there is another star at approx. $2^{\prime \prime}$ in the southwest of MACHO 47.2496.8, the slit was placed perpendicular to this direction. With this instrument setup, the contribution of the close neighbour is negligible.

The reduction of our spectra was performed in the dedicated "UVES context" of the MIDAS environment and included bias correction, cosmic hit correction, flat-fielding, background correction and sky correction. We used optimal extraction to convert frames from pixel-pixel to pixel-order space. The spectra were normalised by dividing the individual orders by a smoothed spline function defined through interactively identified continuum points. For a detailed description of the reduction procedure, we refer to Reyniers (2002). In Table 3, we also list some indicative signal-to-noise values of the final data product. Sample spectra can be found in Figs. 4-6. We note that the most delicate step in the reduction procedure is the normalisation, especially in the blue part of the spectrum, since the spectrum is so crowded that the continuum is seldom reached. The continuum placement is the most important source of uncertainty on the abundances derived from lines in this region.

\section{Abundance analysis}

\subsection{Carbon rich model atmospheres}

Before the actual determination of the atmospheric parameters, a new grid of models for cool carbon-rich stars was calculated with the MARCS code, using opacity sampling in 11000 frequency points. Atomic, diatomic and polyatomic $\left(\mathrm{C}_{2} \mathrm{H}_{2}, \mathrm{HCN}\right.$, and $\mathrm{C}_{3}$ ) opacities were included in addition to the continuous ones in the model atmosphere computations. The models are hydrostatic, spherically symmetric and computed under the assumption of LTE; convective energy transport is included using a Mixing Length Theory (MLT) formulation. Further details on the carbon-star model atmospheres will be given in Jørgensen et al. (2006); the current grid of models is described by Gustafsson et al. (2003). The sub-grid used here was computed for a rather wide range in $T_{\text {eff }}, \log g$, overall metallicity and $\mathrm{C} / \mathrm{O}$ ratios; the stellar mass was set to $1 M_{\odot}$.

\subsection{Atmospheric parameters}

The precise determination of the atmospheric parameters was by no means straightforward. The main difficulty was the lack of existing comparison spectra. Due to the high $\mathrm{C} / \mathrm{O}$ ratio as inferred 
from the strong $\mathrm{C}_{2}$ bands, the spectrum of MACHO 47.2496.8 mimics the spectrum of a cooler carbon star in those spectral regions where molecular lines dominate. The atomic transitions, however, indicate a significantly higher temperature than the typical carbon AGB star temperatures (2500-3500 K). The presence of the molecular lines itself points to a temperature lower than the galactic F-type s-process enriched post-AGB stars.

The final atmospheric parameters were derived by an iterative process of fitting specific spectral regions and fine-tuning the parameters consecutively. These specific regions are carefully chosen in the sense that they contain spectral features strongly depending on the parameters, like lines of different ionisation stages of the same element. Also the wings of the Balmer lines $\mathrm{H} \alpha$ and $\mathrm{H} \beta$ were fitted. The final parameters that were adopted further in the analysis are: $T_{\mathrm{eff}}=4900 \mathrm{~K}, \log g=0.0$, $\xi_{\mathrm{t}}=3.5 \mathrm{~km} \mathrm{~s}^{-1}$ and model metallicity $[\mathrm{M} / \mathrm{H}]=-1.5$. An additional and independent check was made by an equivalent width study of selected iron lines. The search for clean, unblended lines is not easy in the crowded spectrum of MACHO 47.2496.8, but a detailed study of 8 Fe I lines and 5 Fe II nicely confirms the atmospheric parameters as derived by the spectrum synthesis iteration.

The procedure of finding the atmospheric parameters of MACHO 47.2496.8 was also followed in previous analyses of very similar stars (Abia et al. 2002; de Laverny et al. 2006). We refer to the detailed error analyses in those papers for error estimates on the parameters. Here we only remind that the typical errors are as follows: $\pm 250 \mathrm{~K}$ on $T_{\text {eff }}, \pm 0.5$ on $\log g, 1 \mathrm{~km} \mathrm{~s}^{-1}$ on the microturbulent velocity $\xi_{\mathrm{t}}$ and $5 \mathrm{~km} \mathrm{~s}^{-1}$ on the macroturbulent velocity. The latter parameter does not influence the line strengths, but only the line profiles, and is only used to better match synthetic and observed spectra in the spectral syntheses.

\subsection{Spectral regions}

We have analysed in full detail several spectral ranges from $4000 \AA$ to $8000 \AA$. Since the molecular lines of the carbon bearing molecules are comparable in strength to those in carbon stars, we particularly focussed our spectral syntheses on those spectral regions that are also used in the analysis of the cooler galactic carbon stars (see e.g. Abia et al. 2002; de Laverny et al. 2006). For these regions, line lists are carefully composed and these are discussed in Sect. 3 of de Laverny et al. (2006) and will not be repeated here. In general, however, the atomic data of many s-process transitions occuring in the whole spectral region covered by our spectra, are not known to a good precision, which prevents accurate quantitative abundance studies. The most important regions are (i) between $4750 \AA$ and $4950 \AA$ for the s-process elements and the mean metallicity; (ii) 6700-6730 for the Li line; (iii) 4050-4060 $\mathrm{A}$ for $\mathrm{Pb}$; (iv) 4260-4270 $\AA$ for $\mathrm{Tc}$; (v) the $\mathrm{C}_{2}$ band head around $4737 \AA$ for the carbon abundance and isotopic ratio ${ }^{12} \mathrm{C} /{ }^{13} \mathrm{C}$ (vi) the $\mathrm{CN}$ complex between 8000 and $8100 \AA$ for the $\mathrm{N}$ abundance and as an additional tool for the carbon isotopic ratio. Theoretical spectra were computed with the TurboSpectrum code (Alvarez \& Plez 1998; Plez et al. 1992; Plez 1992; Plez et al. 1993) in spherical geometry. The macroturbulence used ranges between 10 and $20 \mathrm{~km} \mathrm{~s}^{-1}$.

The results of our abundance analysis are summarised in Table 4, and are also graphically presented in Fig. 3. The first column in Table 4 gives the solar abundances $\log \epsilon_{\odot}$ of the elements that were studied; the second one contains the actual ions; in the third one the method of abundance determination is given (ss for spectrum synthesis and ew for the equivalent
Table 4. The abundance results for MACHO 47.2496.8. The atmospheric parameters of the model atmosphere are given in Sect. 4.2. The adopted carbon-oxygen difference in the syntheses is $\log (\epsilon(\mathrm{C})-$ $\epsilon(\mathrm{O}))=7.98$. The explanation of the different columns is given in the text (Sect. 4.3). At the bottom, the usual s-process indices, together with metallicity, are given. More information on the calculation of these indices is found in the Sect. 4.7. The elements $\mathrm{C}$ and $\mathrm{O}$ are not separately included in the table due to the specific difficulties for these elements that are discussed in Sect. 4.4.

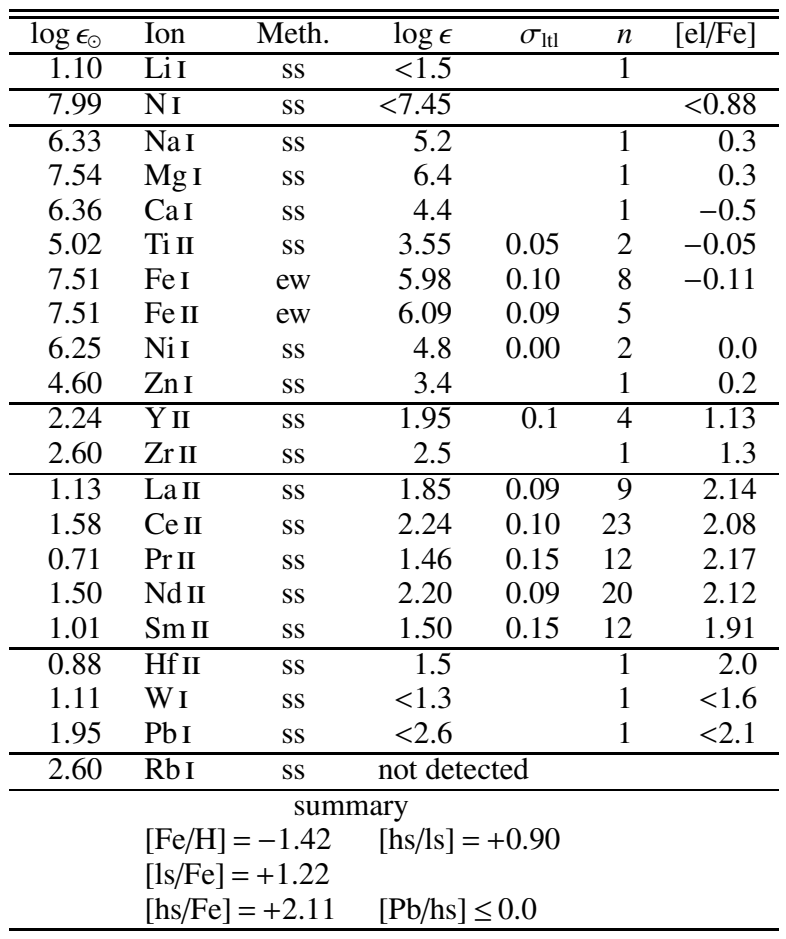

width method); the fourth column gives the absolute abundances derived $\log \epsilon=\log (\mathrm{N}(\mathrm{el}) / \mathrm{N}(\mathrm{H}))+12 ; \sigma_{\mathrm{ltl}}$, the fifth column is the line-to-line scatter of the $n$ lines used (sixth column); the last column gives the abundance relative to iron $[\mathrm{el} / \mathrm{Fe}]$. The solar abundances needed to calculate the $[\mathrm{el} / \mathrm{Fe}]$ values are taken from Grevesse \& Sauval (1998), except: N (Hibbert et al. 1991), Mg (Holweger 2001), La (Lawler et al. 2001). Despite the fact that there are more recent values for some of the solar abundances (especially for the solar CNO), we take these references to ensure as much as possible consistency with our previous analyses, to be able to compare abundance results adequately.

Errors on the reported abundances are difficult to assess, since very different sources can contribute to the total error, and it is often very difficult to perform an adequate error analysis. Main sources of error certainly include continuum placement, undetected molecular and/or atomic features, an uncertain effective temperature and inaccurate $\log (g f)$ values. For error estimates on the reported abundances, we refer again to the error analyses in Abia et al. (2002) and de Laverny et al. (2006). In Fig. 3, typical error bars are drawn, based on the number of lines used: if only one line is used, an error of $0.3 \mathrm{dex}$ is applied, if more than one line is used, an error of 0.2 dex is assumed. The latter error estimate is justified if one assumes that the error on an abundance based on $n$ lines, goes as $\sqrt{\sum_{i} \sigma_{i}^{2}} / n$. In the following subsections, we will briefly discuss our main findings for the different elemental groups. 


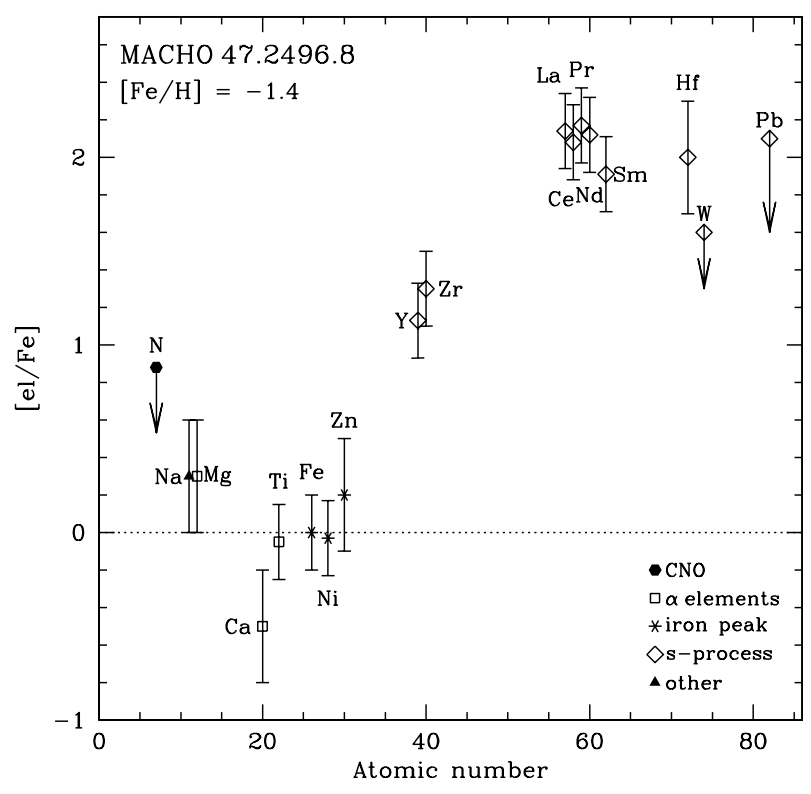

Fig. 3. The abundances of MACHO 47.2496.8 relative to iron [el/Fe].

\section{4. $\mathrm{CNO}$}

Apart from the "classical" atmospheric parameters $T_{\text {eff }}$ and $\log g$, the $\mathrm{C} / \mathrm{O}$ ratio or, more specifically, the difference between the $\mathrm{C}$ and $\mathrm{O}$ absolute abundances, is by far the most important "extra" parameter determining the spectrum of a cool carbon-rich star. Unfortunately, it is impossible to determine this difference accurately, since the $\mathrm{O}$ abundance cannot be determined independently; spectral syntheses are very similar for different $\mathrm{C}$ and $\mathrm{O}$ abundances, as long as the difference $\epsilon(\mathrm{C})-\epsilon(\mathrm{O})$ is kept more or less constant (even within 1 dex of variation of both abundances figures). The reason is that extra $\mathrm{C}$ and $\mathrm{O}$ atoms which are added to the photosphere in similar amount, immediately combine in the strong CO-bond. Therefore, only a lower limit could be derived for the $\mathrm{C} / \mathrm{O}$ ratio: $\mathrm{C} / \mathrm{O}>2$. The carbon isotopic ratio is easier to determine and from the synthesis of the band head region around $4737 \AA$ (Fig. 4), this ratio was found to be ${ }^{12} \mathrm{C} /{ }^{13} \mathrm{C}=200 \pm 25$. The error of \pm 25 indicates the formal error due to model parameters $\left(T_{\text {eff }}, \log g,[\mathrm{M} / \mathrm{H}]\right)$, adding the problem of the continuum location and the uncertainties in the absolute $\mathrm{C}$ and $\mathrm{O}$ abundances.

An upper limit for the nitrogen abundance is derived from the $\mathrm{CN}$ complex at $8000 \AA$, and is found to be $\mathrm{N} / \mathrm{H}<2.8 \times 10^{-5}$ $(\log \epsilon(N)<7.45)$. The spectrum synthesis of this region provides also an additional test for the ${ }^{12} \mathrm{C} /{ }^{13} \mathrm{C}$ ratio. The value of 200 is compatible with the ${ }^{12} \mathrm{CN} /{ }^{13} \mathrm{CN}$ features in the $8000 \AA$ range. The derivation of the carbon isotopic ratio is, however, much more difficult in this $8000 \AA$ region, since the ${ }^{13} \mathrm{CN}$ features are much weaker than in the $4730 \AA$ range, and the uncertainty of the fit is therefore very large.

\subsection{Metallicity and $\alpha$-elements}

The metallicity was already roughly estimated in the iterative process of the model atmosphere parameter determination. A careful synthesis of four clean Fe I lines yields $\log \epsilon(\mathrm{Fe})=6.0$, or $[\mathrm{Fe} / \mathrm{H}]=-1.5$. We also calculated the abundance from 8 unblended FeI lines and 5 unblended Fe II lines through their equivalent width, and the results agree nicely: $\log \epsilon(\mathrm{Fe} \mathrm{I})=5.98$ and $\log \epsilon(\mathrm{Fe}$ II $)=6.09$. We will refer to the latter value as "the"

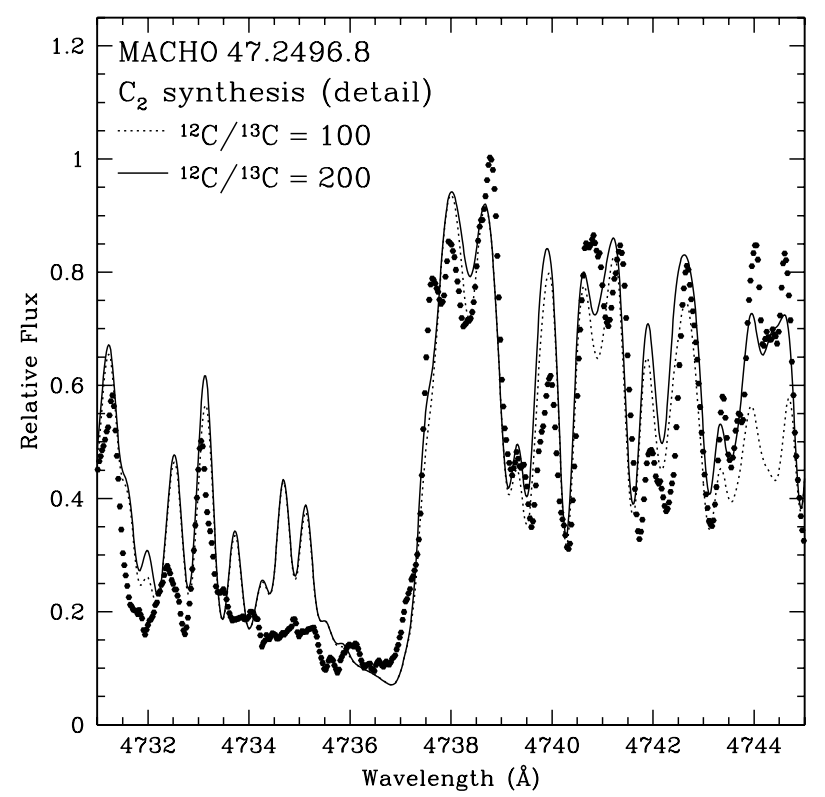

Fig. 4. The VLT-UVES spectrum of MACHO 47.2496.8 (points) around the $\mathrm{C}_{2} 4737 \AA$ band head, overplotted with two spectrum syntheses with a different ${ }^{12} \mathrm{C} /{ }^{13} \mathrm{C}$ isotopic ratio.

metallicity of MACHO 47.2496.8: $[\mathrm{Fe} / \mathrm{H}]=-1.42$. The iron abundance derived from the Fe II-lines is chosen, since also at the relatively low effective temperature of MACHO 47.2496.8, the bulk of the iron atoms in the photosphere is singly ionised. Note that the Fe proxies nickel and zinc nicely follow the iron deficiency.

The $\alpha$-elements show surprisingly different abundances: $\mathrm{Mg}$ seems to be enhanced by 0.3 dex, while $\mathrm{Ca}$ is deficient by approximately 0.5 dex. The reason is not clear, but the difference is likely the initial composition of the object without excluding an observational error due to blending: note that we derive $\mathrm{Mg}$ and $\mathrm{Ca}$ abundances using only one line. The lighter $\alpha$-elements, like $\mathrm{Mg}$, are thought to be mainly produced during the hydrostatic burning of massive stars, while the heavier $\alpha$-elements come from explosive synthesis during SNe II. The abundances of the $\alpha$-elements in MACHO 47.2496.8 are in agreement with the observational trends for the LMC that were recently derived by Pompeia et al. (2006), so there is no evidence for an intrinsic $\alpha$-enrichment or depletion. The same holds for $\mathrm{Na}$. Only $\mathrm{Ca}$ is somewhat lower than expected: Pompeia et al. (2006) predict $[\mathrm{Ca} / \mathrm{Fe}] \sim 0$ for a metallicity of $[\mathrm{Fe} / \mathrm{H}]=-1.4$, while we derived $[\mathrm{Ca} / \mathrm{Fe}]=-0.5$ for MACHO 47.2496.8.

\subsection{Lithium}

Although the region around the lithium doublet at $6708 \AA$ is not very well fitted, there is no evidence for an enhanced $\mathrm{Li}$ abundance. The upper limit derived for $\mathrm{Li}$ is $\log \epsilon(\mathrm{Li})<1.5$. Note that the $\mathrm{Li}$ abundance for a strongly s-process enriched object is difficult to determine, since the Li doublet is strongly blended with a Ce II transition at 6708.10 ̊ (Reyniers et al. 2002).

\subsection{S-process elements}

Although trace elements by nature, the absolute dominance of transitions of s-process elements in the spectra is overwhelming. This is illustrated in Fig. 5, in which the synthesis is performed with and without s-process enhancement. S-process elements are 


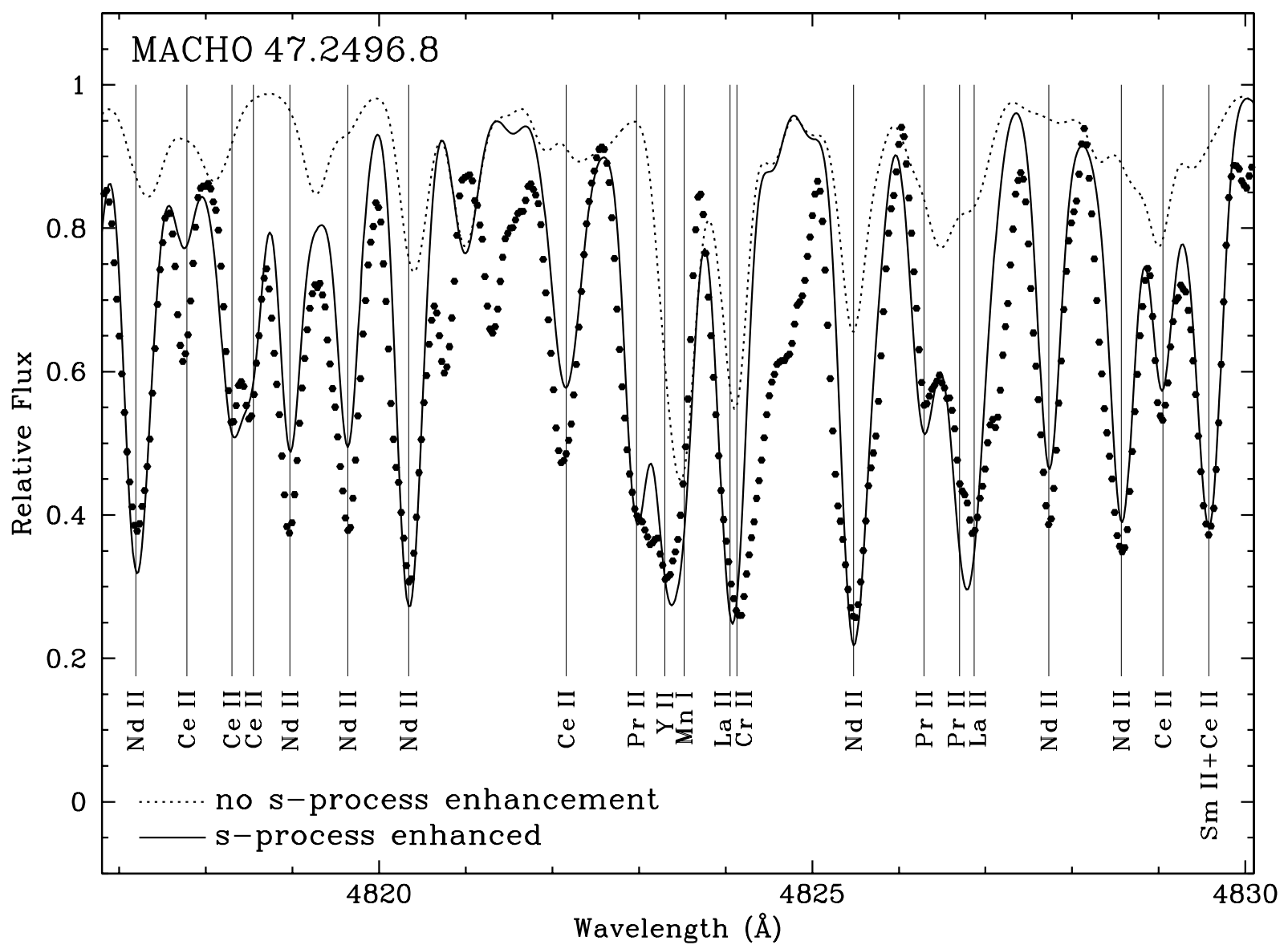

Fig. 5. The VLT-UVES spectrum of MACHO 47.2496 .8 (points) overplotted with two spectrum syntheses. Both syntheses are made with the same model atmosphere, only the abundances of the s-process elements differ. The synthesis in the dotted line is made with solar abundances for the s-process elements (obviously scaled down to the metallicity of MACHO 47.2496.8), while the synthesis in the full line is made with the enhanced s-process abundances as tabulated in Table 4 . The observed spectrum is very well reproduced by the spectrum synthesis, although there are clearly still some lines missing in the line lists.

classically divided into the light ones noted ls ( $\mathrm{Sr}, \mathrm{Y}$ and $\mathrm{Zr}$ ) and the heavy ones, noted hs (Ba, La, Ce, Pr, Nd and Sm). Some elements show a wealth of atomic transitions in the optical, like $\mathrm{Ce}$ and $\mathrm{Nd}$, and the abundances of these elements can be derived with great accuracy. Others, like $\mathrm{Sr}$ and $\mathrm{Ba}$, only produce a few strong resonance lines that are not suitable for abundance determination purposes. This fact should be taken into account when comparing individual s-process abundances. We could also derive an abundance for the trans-Ba element hafnium (Hf), but it is based on only one line, so it should be treated with caution.

We have also studied two resonance lines of Ba II to estimate the $\mathrm{Ba}$ abundance. On top of being strongly saturated, the profiles are broader than other atomic lines and both lines show a blue-shifted component (14.6 and $15.5 \mathrm{~km} \mathrm{~s}^{-1}$, respectively). This is probably due to the dynamical structure of the outer atmosphere of this pulsating star. Using only the component at the same photospheric velocity as the other atomic lines, we derive an upper limit of $\log \epsilon(\mathrm{Ba})<2.8$. We conclude, however, that the special line profile makes the $\mathrm{Ba}$ abundance very uncertain.

All s-process abundances are based on lines of singly ionised species. Additionally, we have also searched for lines of doubly ionised $\mathrm{Pr}$ and $\mathrm{Nd}$, which are known to produce some strong lines in the spectra of enriched post-AGB stars (e.g. Reyniers et al. 2004). We found one Nd III line at 5294.099 $\AA$ and two lines of Pr III (at 5284.693 $\AA$ and 5998.930 ̊) suitable for abundance determination. The Nd III line yields the same abundance as the one from the $\mathrm{Nd}$ II lines reported in Table 4; the two Pr III lines yield an abundance of $\log \epsilon(\operatorname{Pr})=1.85$ and 2.26 respectively, and are much less consistent with their singly ionised counterparts. It is not clear why the discrepancy $(\sim 0.6 \mathrm{dex})$ is so large.

In order to characterize the s-process pattern, and to be able to adequately compare the observed pattern with other sprocess enriched stars, three s-process indices are usually defined: [ls/Fe], [hs/Fe] and [hs/ls]. Which specific elements are taken into account to determine these indices, are, unfortunately, author-dependent, and are mainly just determined by the elemental abundances that are obtained in the analysis. Here, we define the ls-index as the mean of $\mathrm{Y}$ and $\mathrm{Zr}$; the hs-index as the mean of La, Ce and $\mathrm{Nd}$; and obviously [hs/ls]=[hs/Fe]-[ls/Fe]. The elements $\mathrm{Pr}$ and $\mathrm{Sm}$ are excluded from the hs-index to be able to compare with other s-process enriched stars. Indeed, in many of the s-process studies, abundances for these elements are lacking, and hence they are not incorporated in the indices. The resulting indices are given at the bottom of Table 4 .

\subsection{Technetium}

The presence of technetium (Tc, $\mathrm{Z}=43$ ) is a well known indicator that a star is currently undergoing dredge-ups on the AGB phase, or that it has just left this phase, since the half-life of ${ }^{99} \mathrm{Tc}$ is $\sim 2 \times 10^{5} \mathrm{yr}$. The detection of Tc is, however, complicated since 
all available lines are quite weak, and they are often blended by much stronger atomic and/or molecular lines. One of the lines that is often used in abundance studies of AGB stars, is the resonance line at $4262.27 \AA$. The continuum placement is the main source of uncertainty in this crowded spectral region. Moreover, at the effective temperature of MACHO 47.2496.8, the Tc line is intrinsically too weak to see a clear difference in the synthesized line for different abundances. We have to conclude that Tc cannot be used for MACHO 47.2496.8 as a criterion for the intrinsic enrichment.

\subsection{Niobium}

Besides Tc, there is another element whose abundance can be used as a criterion to determine whether the star is intrinsically or extrinsically enriched: niobium. $\mathrm{Nb}$, which has only one stable isotope, is exclusively produced by the decay of ${ }^{93} \mathrm{Zr}$ (half-life $\tau_{1 / 2} \sim 1.5 \times 10^{6} \mathrm{yr}$ ) and therefore $\mathrm{Nb}$ populates the stellar surface only million years after the s-process elements have been dredged-up. As a consequence, intrinsically (post-)AGB stars have a $\mathrm{Nb}$ abundance much lower than extrinsically enriched stars for which $[\mathrm{Nb} / \mathrm{Zr}] \simeq 0$. For intrinsically enriched stars, the $[\mathrm{Nb} / \mathrm{Zr}]$ abundance depends only very slightly on the metallicity, and is predicted to be $[\mathrm{Nb} / \mathrm{Zr}] \simeq-1.0$ (Bisterzo et al. 2006). For MACHO 47.2496.8, we checked all available Nb I and Nb II lines in the spectrum, but unfortunately none of them is suitable to derive a reliable abundance or upper limit, since they are all situated in the blue part of the spectrum for which the molecular veiling is most severe. There is one Nb II line at $4789.89 \AA$ that could give an estimate for the upper limit. Again, the continuum position is the main source of uncertainty in this spectral region. Also, the blend in the immediate vicinity of the $\mathrm{Nb}$ line at $\sim 4790.5 \AA$ is clearly lacking in our line list. The derived upper limit of $[\mathrm{Nb} / \mathrm{Zr}] \leq-0.3$ is therefore very uncertain, but points rather to an intrinsic than an extrinsic nature. Spectra taken in a hotter phase might be more suitable to study this $\mathrm{Nb}$ line in more detail.

\subsection{Lead}

The end point of the s-process path is situated at lead and bismuth. Since the discovery of the so-called "lead stars" by Van Eck et al. (2001), lead has become a very important issue in the study of the s-process. Unfortunately, lead has only a few lines in the optical region, and they are all situated in crowded regions in the blue spectral domain. In MACHO 47.2496.8, only the line at $4057.81 \AA$ is accessible, and even there the blending by molecular lines and s-process elements is severe. As a consequence, the main source of uncertainty of the $\mathrm{Pb}$ synthesis in this region is the continuum placement. Therefore, we can only derive an upper limit for the $\mathrm{Pb}$ abundance: $\log \epsilon(\mathrm{Pb})<2.8$, as illustrated in Fig. 6.

\section{Discussion: intrinsic or extrinsic?}

Our abundance analysis (Table 4 and Fig. 3) clearly shows that MACHO 47.2496.8 is a metal poor, but strongly s-process and carbon enriched object in the LMC. Contrary to Galactic RV Tauri stars (see Sect. 1), there is no evidence for depletion of refractory elements. In a carbon-rich $(\mathrm{C} / \mathrm{O}>1)$ environment, $\mathrm{ZrC}$ is likely to condense first (Lodders \& Fegley 1999) followed by TiC and SiC. For MACHO 47.2496.8, the abundances of these elements follow the trend which is expected from a

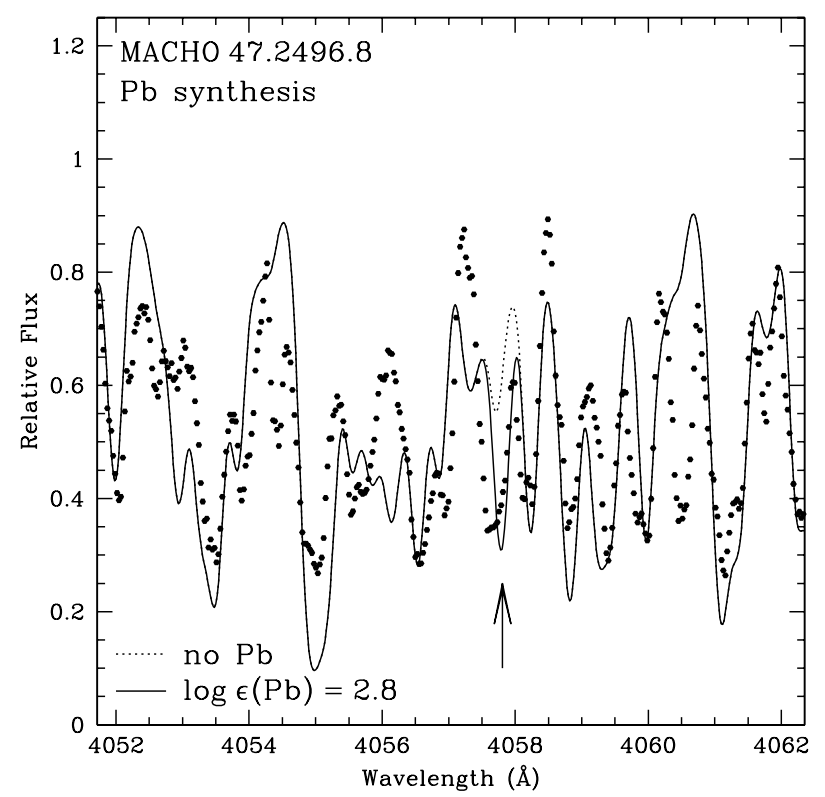

Fig. 6. Synthesis of the $\mathrm{Pb}$ I line at $4057.81 \AA$. The exact position of the continuum is far from clear in the vicinity of the line, which makes it only possible to derive an upper limit for the $\mathrm{Pb}$ abundance.

star that underwent an efficient dredge-up while evolving on the AGB, so no depletion has taken place. To determine whether this enrichment is intrinsic or extrinsic is not straightforward but in the following we argue in favour for the intrinsic interpretation.

\subsection{Abundances}

From Sect. 4.8 it is clear that the spectral feature, Tc, that is classically used to determine whether a star is intrinsically or extrinsically enriched, cannot be used for MACHO 47.2496.8, mainly due to its relatively high $T_{\text {eff }}$. An alternative indicator, the $[\mathrm{Nb} / \mathrm{Zr}]$ abundance discussed in 4.9 , is also difficult to quantify, but might have some potential for spectra that are taken in a hotter phase.

\subsection{Carbon star luminosity function}

Since carbon star counts in the Magellanic Clouds are considered to be nearly complete, the luminosity functions for the carbon stars (CSLF) in the Clouds can be constructed. In the LMC, 7750 carbon stars are detected, and a histogram of the observational CSLF can be found in e.g. Fig. 6 of Groenewegen (2006). This histogram does not indicate a clear faint limit to the luminosity function of the N-type carbon stars. The reproduction of the CSLF of the LMC by detailed nucleosynthetic models is a long standing problem, since these models do not succeed in producing the carbon stars at the low luminosity end of the CSLF. Recently, it has been argued that the low-luminosity end of the CSLF is populated by first giant-branch stars that are enriched by mass transfer from a former AGB companion. This idea was quantitatively worked out in Izzard \& Tout (2004). In this paper, the transition between an extrinsically enriched carbon star and a genuine AGB carbon star is situated around $M_{\text {bol }} \sim-4$. Consequently, the bolometric magnitude $M_{\text {bol }} \simeq-4.5$ of MACHO 47.2496.8, inferred in Sect. 2, does not provide a strong constraint whether the object is either intrinsically or extrinsically enriched. 
We compared the flux of our program star to the flux of the $\mathrm{N}$ stars by integrating over the wavebands B to K. The mean figure for MACHO 47.2496.8 has been calculated assuming that the ratio between maximum and intensity-mean light, calculated for $V$ and $R$ using the intensity-scaled light curves from the MACHO website, applies to all wavelengths. This is a conservative assumption, as the amplitude is probably less in the near infrared. The relative flux in $B V R I$ for the $\mathrm{N}$ stars has been assumed to be the same as for galactic $\mathrm{N}$ stars, using the data of Walker (1979). Fluxes have been calculated in magnitudes on an arbitrary scale, and show that MACHO 47.2496.8 is 0.09 mag (at minimum light) and $0.30 \mathrm{mag}$ (at maximum light) brighter than the faint limit for $\mathrm{N}$ stars in the LMC.

It is clear that MACHO 47.2496.8 is located at the low luminosity end of the carbon star luminosity function of the LMC. Its metallicity is, however, quite low compared to the bulk of the LMC stars. A better luminosity function may therefore come from the SMC where it is well known that the CSLF is shifted to lower luminosities (Groenewegen 1999). The low intrinsic metallicity therefore favours even more the intrinsic nature of the s-process enrichment. In fact, at the metallicity of MACHO $47.2496 .8\left(\sim Z_{\odot} / 30\right)$, the minimum luminosity for the formation of an AGB carbon star may be slightly lower than $M_{\text {bol }} \sim-4.0$, the exact value depending on the mass-loss parametrization (e.g. Straniero et al. 2003).

\subsection{Comparison with galactic $\mathrm{CH}$ stars}

$\mathrm{CH}$-stars are metal poor, carbon rich giants with spectral types from $\mathrm{G}$ to $\mathrm{K}$, with enhanced $\mathrm{CH}, \mathrm{C}_{2}$ and s-process elements relative to normal giants. $\mathrm{CH}$-stars have typically also large radial velocities of $\sim 100 \mathrm{~km} \mathrm{~s}^{-1}$. It is likely that all CH-stars are binaries in which the present primary has gained processed material from a formerly-AGB companion, now a white dwarf (McClure $\&$ Woodsworth 1990). The first accurate quantitative abundance analyses were done by Vanture (1992a,b,c). He found that the s-process abundance pattern of the $\mathrm{CH}$-stars is characterised by large s-process enhancements and large [hs/ls] ratios of $>+0.9$. Their metal deficiency, carbon enrichment and s-process pattern are similar to MACHO 47.2496.8, and they are therefore ideal galactic comparison stars. The majority of $\mathrm{CH}$-giants, however, show very low carbon isotopic ratios, typically ${ }^{12} \mathrm{C} /{ }^{13} \mathrm{C}<10$ (Vanture 1992a), which is not seen in MACHO 47.2496.8.

More recent abundance analyses of $\mathrm{CH}$-stars (Van Eck et al. 2001; Van Eck et al. 2003; Aoki et al. 2001, 2002) confirmed the older results of Vanture (1992c), and focussed on the detection of an enhanced lead content, which was predicted in different nucleosynthetic AGB models (e.g. Gallino et al. 1998; Goriely \& Mowlavi 2000). As first discovered by Van Eck et al. (2001), a subgroup of the $\mathrm{CH}$-stars does show this predicted $\mathrm{Pb}$ enhancement (typically $[\mathrm{Pb} / \mathrm{hs}] \simeq+1$ ), but there are also $\mathrm{CH}$-stars that are not compatible with the predictions, showing $[\mathrm{Pb} / \mathrm{hs}]$ ratios that are more than one magnitude lower than predicted (see e.g. Fig. 4 in Van Eck et al. 2003).

The $\mathrm{Pb}$ abundance of MACHO 47.2496.8 is very uncertain, and in Sect. 4.10, we were only able to derive an upper limit of $[\mathrm{Pb} / \mathrm{hs}] \sim 0$. Therefore MACHO 47.2496.8 is considered as a "lead-low" star. Nevertheless, the low $[\mathrm{Pb} / \mathrm{hs}]$ ratio as well as the other s-process indices are still compatible with recent nucleosynthesis calculations in low-mass AGB stars (Gallino \& Bisterzo 2006).

\section{Conclusion}

In this paper, we have presented our study of the brightest RV Tauri star that was found in the MACHO experiment: MACHO 47.2496.8 (Alcock et al. 1998). The star is strongly pulsating with a peak-to-peak amplitude in $V$ of $\sim 1.4$ mag. A SED constructed using recent CCD photometry taken with the Euler telescope at La Silla, indicates a reddening of $E(B-V)=$ 0.44 , while the interstellar reddening towards this region in the LMC is $E(B-V)=0.11$. The intrinsic bolometric magnitude $M_{\text {bol }}$ calculated from the spectral energy distribution is -4.5 , placing the star in the low luminosity end of the carbon star luminosity function of the LMC.

Our high-resolution, high signal-to-noise optical UVES spectra are unfortunately taken in a cool phase. The main results of our detailed abundance analysis performed by a careful synthesis of some regions that are already well studied in galactic carbon stars, are:

- a surprisingly low metallicity: $[\mathrm{Fe} / \mathrm{H}]=-1.4$, with no evidence of depletion;

- a high C/O ratio, certainly larger than 2 , and a carbon isotopic ratio ${ }^{12} \mathrm{C} /{ }^{13} \mathrm{C}$ of around 200 ;

- a large s-process enrichment with $[1 \mathrm{~s} / \mathrm{Fe}]=+1.2$, and $[\mathrm{hs} / \mathrm{Fe}]=+2.1$;

- a low $\mathrm{Pb}$ abundance $[\mathrm{Pb} / \mathrm{Fe}] \simeq[\mathrm{hs} / \mathrm{Fe}]$.

We used a new grid of state-of-the-art MARCS models for cool carbon rich stars, which was especially constructed for this analysis.

Although we cannot totally exclude that the star is extrinsically enriched by a former AGB companion which should be seen now as a white dwarf, the enrichment is likely of an intrinsic origin. The luminosity, although quite low, is still compatible with an advanced phase of a very low-mass star, and the pulsations are prototypical of an RV Tauri like object. Unfortunately, the star is too hot to use technetium as a criterion of an in situ enrichment. Also the alternative element suitable as an intrinsic/extrinsic test, niobium, cannot be used for this star. New spectra, taken in a hotter phase when the molecular veiling is absent, could enable a conclusive test concerning the intrinsic/extrinsic dilemma.

With its low luminosity and certainly very low inital metallicity, MACHO 47.2496.8 represents the final evolution of a star which must have had an initial mass very close to solar. To our knowledge, this is the first detailed chemical analysis of a postAGB star with a known distance and accurate luminosity estimate. The large carbon enhancement and the very rich s-process nucleosynthesis show that also very low-mass objects will undergo strong chemical changes during AGB evolution.

Acknowledgements. It is a pleasure to thank Maria Lugaro and Axel Bonačić Marinović, who were immediately willing to test their latest models on our results, which initiated a most promising collaboration. The authors would also like to thank Oscar Straniero for the Nb prediction, Pieter Deroo for the help with the construction of the SED, Sophie Saesen for the Euler observations and the anonymous referee for the many useful comments that improved the paper considerably. The Geneva staff is thanked for observation time on the Euler telescope. This paper utilizes public domain data obtained by the MACHO Project, and data from the Vienna Atomic Line Database. M.R. and L.D. acknowledge financial support from the Fund for Scientific Research - Flanders (Belgium); C.A. has been partially supported by the Spanish grant AYA2005-08013-C0303; K.E. thankfully acknowledges support by the Swedish Research Council. 


\section{References}

Abia, C., Domínguez, I., Gallino, R., et al. 2002, ApJ, 579, 817

Alcock, C., Allsman, R. A., Alves, D. R., et al. 1998, AJ, 115, 1921

Alcock, C., Allsman, R. A., Alves, D. R., et al. 1999, PASP, 111, 1539

Alcock, C., Axelrod, T. S., Bennett, D. P., et al. 1992, in Robotic Telescopes in the 1990s, ASP Conf. Ser., 34, 193

Alcock, C., Allsman, R. A., Axelrod, T. S., et al. 1995, AJ, 109, 1653

Alvarez, R., \& Plez, B. 1998, A\&A, 330, 1109

Aoki, W., Ryan, S. G., Norris, J. E., et al. 2001, ApJ, 561, 346

Aoki, W., Ryan, S. G., Norris, J. E., et al. 2002, ApJ, 580, 1149

Ardeberg, A., Brunet, J. P., Maurice, E., \& Prevot, L. 1972, A\&AS, 6, 249

Bisterzo, S., Gallino, R., Straniero, O., et al. 2006, Mem. Soc. Astr. Ita., in press

Conti, P. S., Garmany, C. D., \& Massey, P. 1986, AJ, 92, 48

Crampton, D. 1979, ApJ, 230, 717

de Laverny, P., Abia, C., Domínguez, I., et al. 2006, A\&A, 446, 1107

De Ruyter, S., van Winckel, H., Maas, T., et al. 2006, A\&A, 448, 641

Deroo, P., Reyniers, M., Van Winckel, H., Goriely, S., \& Siess, L. 2005, A\&A, 438, 987

Fitzpatrick, E. L. 1988, ApJ, 335, 703

Fitzpatrick, E. L., \& Garmany, C. D. 1990, ApJ, 363, 119

Fouqué, P., Chevallier, L., Cohen, M., et al. 2000, A\&AS, 141, 313

Gallino, R., Arlandini, C., Busso, M., et al. 1998, ApJ, 497, 388

Gallino, R., \& Bisterzo, S. 2006, XIII Nuclear Astrophysics Workshop, ed. E. Müller, T. Janka, \& W. Hillebrant, in press

Giridhar, S., Lambert, D. L., Reddy, B. E., Gonzalez, G., \& Yong, D. 2005, ApJ, 627,432

Glass, I. S. 1973, MNRAS, 164, 155

Goriely, S., \& Mowlavi, N. 2000, A\&A, 362, 599

Grevesse, N., \& Sauval, A. J. 1998, Space Sci. Rev., 85, 161

Groenewegen, M. A. T. 1999, in Asymptotic Giant Branch Stars, ed. T. Le Bertre, A. Lebre, \& C. Waelkens, IAU Symp., 191, 535

Groenewegen, M. A. T. 2006, in proceedings of the ESO Workshop Planetary Nebulae beyond the Milky Way, ed. L. Stanghellini, J. Walsh, \& N. G. Douglas, 108

Gustafsson, B., Edvardsson, B., Eriksson, K., et al. 2003, in Stellar Atmosphere Modeling, ed. I. Hubeny, D. Mihalas, \& K. Werner, ASP Conf. Ser., 288, 331 Harmanec, P., \& Božić, H. 2001, A\&A, 369, 1140

Hibbert, A., Biemont, E., Godefroid, M., \& Vaeck, N. 1991, A\&AS, 88, 505

Holweger, H. 2001, in Joint SOHO/ACE workshop Solar and Galactic Composition, ed. R. F. Wimmer-Schweingruber, AIP Conf. Proc., 598, 23

Isserstedt, J. 1975, A\&AS, 19, 259

Isserstedt, J. 1979, A\&AS, 38, 239
Isserstedt, J. 1982, A\&AS, 50, 7

Izzard, R. G., \& Tout, C. A. 2004, MNRAS, 350, L1

Jørgensen, U., et al. 2006, A\&A, in preparation

Jura, M. 1986, ApJ, 309, 732

Keller, S. C., Bessell, M. S., Cook, K. H., Geha, M., \& Syphers, D. 2002, AJ, 124,2039

Lawler, J. E., Bonvallet, G., \& Sneden, C. 2001, ApJ, 556, 452

Lloyd Evans, T., \& Pollard, K. R. 2004, in Variable Stars in the Local Group, ed.

D. W. Kurtz, \& K. R. Pollard, IAU Colloq., 193, ASP Conf. Ser., 310, 344

Lodders, K., \& Fegley, B. 1999, in Asymptotic Giant Branch Stars, ed. T. Le Bertre, A. Lebre, \& C. Waelkens, IAU Symp., 191, 279

Maas, T., Van Winckel, H., \& Lloyd Evans, T. 2005, A\&A, 429, 297

McClure, R. D., \& Woodsworth, A. W. 1990, ApJ, 352, 709

Plez, B. 1992, A\&AS, 94, 527

Plez, B., Brett, J. M., \& Nordlund, A. 1992, A\&A, 256, 551

Plez, B., Smith, V. V., \& Lambert, D. L. 1993, ApJ, 418, 812

Pollard, K. R., Cottrell, P. L., Kilmartin, P. M., \& Gilmore, A. C. 1996, MNRAS, 279, 949

Pollard, K. R., \& Lloyd Evans, T. 2000, AJ, 120, 3098

Pompeia, L., Hill, V., Spite, M., et al. 2006 [arXiv: astro-ph/0604009]

Reyniers, M. 2002, Ph.D. Thesis, K.U. Leuven

Reyniers, M., Van Winckel, H., Biémont, E., \& Quinet, P. 2002, A\&A, 395, L35

Reyniers, M., Van Winckel, H., Gallino, R., \& Straniero, O. 2004, A\&A, 417, 269

Rousseau, J., Martin, N., Prevot, L., et al. 1978, A\&AS, 31, 243

Schmidt-Kaler, T., Gochermann, J., Oestreicher, M. O., et al. 1999, MNRAS, 306,279

Schombert, J. M., Wallin, J. F. \& Struck-Marcell, C. 1990, AJ, 99, 497

Stasińska, G., Szczerba, R., Schmidt, M., \& Siódmiak, N. 2006, A\&A, 450, 701 Stellingwerf, R. F. 1978, ApJ, 224, 953

Straniero, O., Domínguez, I., Cristallo, R., \& Gallino, R. 2003, Publications of the Astronomical Society of Australia, 20, 389

Van Eck, S., Goriely, S., Jorissen, A., \& Plez, B. 2001, Nature, 412, 793

Van Eck, S., Goriely, S., Jorissen, A., \& Plez, B. 2003, A\&A, 404, 291

Van Winckel, H. 2003, ARA\&A, 41, 391

Vanture, A. D. 1992a, AJ, 103, 2035

Vanture, A. D. 1992b, AJ, 104, 1986

Vanture, A. D. 1992c, AJ, 104, 1997

Walker, A. R. 1979, South African Astronomical Observatory Circular, 1, 112

Waters, L. B. F. M., Trams, N. R., \& Waelkens, C. 1992, A\&A, 262, L37

Wood, P. R., Alcock, C., Allsman, R. A., et al. 1999, in Asymptotic Giant Branch Stars, IAU Symp., 191, 151

Zaritsky, D., Harris, J., Thompson, I. B., \& Grebel, E. K. 2004, AJ, 128, 1606 